\title{
Proyectos de infraestructura de transporte y su relación con el desarrollo de las localidades
}

\author{
Mauricio Casanova \\ Universidad de Chile
}

\begin{abstract}
Resumen
Mediante una aproximación que mezcla análisis cuantitativo y cualitativo, de terreno, ex-post, experimental, de naturaleza meso-económica, no exhaustiva y específica a cada territorio, se busca determinar si el desarrollo territorial esperado producto de mejoras en las condiciones de conectividad en Chile (medido a través de la inversión pública en infraestructura de transporte) se ha materializado efectivamente, si ello es percibido así por la ciudadanía, y cuáles factores explican dichos resultados. Para lo anterior, se analizan indicadores de cambio o evolución de pobreza, mercado laboral, competitividad y población, a nivel comunal; y luego se indaga en estas dimensiones a través de entrevistas en profundidad a personas de seis comunas con alta inversión en infraestructura. La principal conclusión del análisis es que existe escasa relación entre las mejoras en conectividad de transporte y los cambios observados y percibidos en el nivel de desarrollo socio-económico comunal, a pesar de que las personas opinan que dicha relación efectivamente existe. Esto parece ser la manifestación de una falla en la política pública de conectividad, en tanto no ha generado los resultados esperados. La causa de lo anterior estaría en la inadecuada definición de sus objetivos, y la incorrecta identificación de los beneficios y beneficiarios.

Palabras clave: Desarrollo territorial, inversión pública en infraestructura de transporte, indicadores socioeconómicos.
\end{abstract}

Transport Infrastructure Projects and its Relationship with Localities Development

\begin{abstract}
This article seeks to determine whether the territorial development expected product of improvements in connectivity conditions in Chile (measured through public investment in transport infrastructure) has actually materialized, if it is well received by the public, and what factors explain such results. The study was conducted using an approach that combines quantitative and qualitative analysis, field analysis, ex-post, experimental, meso-economic nature, not exhaustive and specific to each territory. For this, indicators of change or evolution of poverty, labor market, competitiveness and population are analyzed at community level. Then, the study explores these dimensions through in-depth interviews people in six communes with high investment in infrastructure. The main conclusion of the analysis is that there is little relationship between improvements in transport connectivity and observed changes in the perceived level of community socio-economic development, even though people think that such relationship exist. This seems to be the manifestation of the mistake in connectivity public policy because it has not generated the expected results. The cause of this would be the inadequate definition of their objectives, and the incorrect identification of benefits and beneficiaries.
\end{abstract}

Keywords: Territorial development, public investment in transport infrastructure, socio-economic indicators.

*Dirección de correspondencia [Correspondence address]:

Mauricio Casanova, Universidad de Chile

E-mail: mcasanova@mtt.gob.cl

\section{Proyectos de infraestructura de transporte y su relación con el desarrollo de las localidades}

El presente trabajo busca indagar en los resultados que han tenido las políticas públicas de provisión de infraestructura de transporte sobre el desarrollo de las localidades, y razonar sobre cuáles serían los factores que parecen influir para que las 
iniciativas de acceso a un territorio aporten a generar un círculo virtuoso de desarrollo.

Tal como indica un informe de la OECD (2002), el concepto de accesibilidad está relacionado con aumentar la posibilidad de viajar, por la vía de la reducción del tiempo, el costo del viaje u otras barreras, lo que finalmente "aumenta la facilidad con la que una actividad social o económica puede ser alcanzada utilizando el sistema de transporte" (OCDE, 2002:38); en otras palabras, la accesibilidad está relacionada con "la facilidad de acceder [...] a las oportunidades" (Litman, 2014).

La política pública de accesibilidad interna del territorio chileno, es provista principalmente por el Ministerio de Obras Públicas (MOP) y el Ministerio de Transportes y Telecomunicaciones (MTT). La primera Secretaría de Estado es la encargada de todas las etapas necesarias para la materialización y explotación de los caminos públicos, aeropuertos, puertos y muelles, entre otros. Por su parte el MTT es el organismo encargado de proponer las políticas en materia de tránsito por calles y caminos y demás vías públicas.

En Chile se han evaluado los proyectos de infraestructura mediante análisis costo beneficio, con las herramientas propias de la evaluación económica de proyectos", las que presentan "serios problemas cuando el sentido de un proyecto es social y no netamente económico. Esto porque la cuantificación de los beneficios requiere de una consecuente estimación de demanda, y de las ventajas que significaría la construcción de la obra" (Ministerio de Obras Públicas, 2004:10).

Es por esta razón que este estudio de caso busca explorar la relación entre las mejoras en infraestructura de transporte y el desarrollo socio-económico que las localidades conectadas mostraron en Chile, durante el período 1992-2012. Se busca, además, identificar brechas y determinar cuáles son los factores que influyen para que las iniciativas de acceso a un territorio aporten a la generación de un círculo virtuoso de desarrollo.

\section{Relaciones entre infraestructura de transporte, crecimiento y desarrollo}

La relación entre el transporte y las actividades sociales y económicas que se desarrollan en su entorno, se manifiesta, según Vickerman (2007), entre otros autores, en la interrelación con la localización

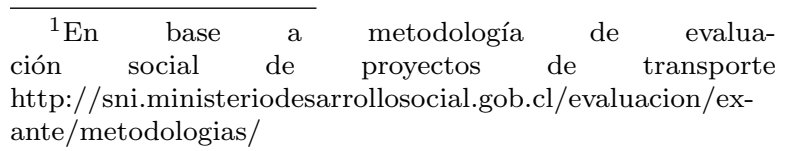

Rev. Est. de Políticas Públicas, 2016, vol. 3 (junio) de actividades comerciales, residencias, lugares de trabajo, y servicios públicos, entre otros. La localización deriva, a su vez, del valor relativo que los diversos actores le otorgan a los lugares o a la posibilidad de acceder a dichos lugares (Litman, 2014) en relación con el costo y tiempo del viaje. No se trata sólo del comportamiento de los agentes del mercado, si no que cómo sus actividades se organizan. Esas relaciones no ocurren de manera independiente y los sistemas de actividades y el transporte se afectan mutuamente (Ortúzar y Willumsen, 2008; Ministerio de Obras Públicas, 2014; Vickerman, 2007).

En general, el espacio importa y moldea el potencial de desarrollo no sólo del territorio, sino que también de los individuos que viven en ellos. En consecuencia, si se quiere influir en el desarrollo, las estrategias no deben ser espacialmente neutras (space-neutral), sino que basadas en lo local (placebased) y altamente contingente al contexto (Barca et al., 2011).

Las políticas territorialmente ciegas asumieron que por vía de promover la movilidad, se obtendrían territorios con una distribución homogénea de bienestar y la convergencia de zonas rezagadas (Barca et al., 2011). Sin embargo, se han manifestado efectos negativos que, en teoría, sería posible observar en el caso de materializarse más infraestructura de transporte en un territorio o entre dos territorios con condiciones disímiles. Según indica OECD (2002) no es claro si las políticas de accesibilidad proveen mayores beneficios a las regiones pobres o si inducirán desarrollo económico en el largo plazo. Se menciona además, que existe poca evidencia empírica para saber si la inversión en infraestructura a nivel regional en efecto fortalece al receptor, dado que expande el mercado y permite la migración de trabajadores a otros centros donde las oportunidades percibidas son mayores. Adicionalmente, indica el informe, el desarrollo se puede dar en varias dimensiones (económica, social o ambiental) y es difícil sopesarlas mutuamente.

Yendo más allá, incluso se ha reportado alguna evidencia sobre una relación negativa entre stock de infraestructura de transporte de un territorio y la producción de otro (Moreno y López-Bazo, 2003) (efecto de spillover negativo). La causa estaría en el hecho de que cuando los factores productivos son móviles, la infraestructura de transporte en una región puede extraer la producción industrial de otra. Puesto de otra manera, regiones con infraestructura similar compiten por los factores móviles de producción. Esto genera que en las primeras etapas de una política pública para fortalecer la integración, se podrían ver incrementadas las desigualdades (Moreno y López-Bazo, 2003). 
Una consecuencia de lo anterior es que las regiones podrían utilizar la infraestructura como una herramienta para atraer factores productivos incrementando su propia producción industrial a expensas de las otras regiones. En consecuencia, si las regiones tomasen la decisión respecto de las inversiones, podría significar un exceso de infraestructura.

En Chile existe alguna evidencia de problemas. El informe de la OECD sobre Desarrollo Territorial del año 2008 indica que la construcción de infraestructura para la conectividad se ha concentrado en la zona central o en torno a las grandes ciudades y sugiere que una estrategia de planificación que refuerce el nivel regional es el cambio que se debe dar para continuar en la senda de desarrollo, pues, indica, a diferencia del nivel central, el regional es "fundamental para la discusión, planificación y coordinación de las políticas de desarrollo del transporte" (Subsecretaría de Desarrollo Regional y Administrativo, 2009).

Una publicación algo anterior de la CEPAL sugiere que las realidades locales influyen fuertemente en el nivel del impacto de las medidas de conectividad y que "lo local cobra importancia frente a la macro red". Parte de dicha relevancia se observaría, según dicha publicación, en el componente institucional, el que se releva "al entender que la red física se despliega sobre el espacio, donde subyacen ordenamientos y administraciones intangibles que organizan el territorio" (Figueroa y Rozas, 2005). La infraestructura de transporte es, más que una red, la base sobre la que se despliegan servicios que permiten acrecentar la eficacia de la coordinación de los agentes en el espacio (Joignaux y Langumier, 2004), reforzando la idea de calidad y nivel de servicio al evaluar la infraestructura tomando en cuenta la compleja interrelación entre los agentes.

\section{Efectos socio-económicos de la infra- estructura de transporte}

Los efectos de la inversión en infraestructura de transporte se pueden clasificar en directos, siendo los principales el tiempo de viaje, los costos operativos de los vehículos y la seguridad (Vickerman, 2007); y efectos socio-económicos amplios (OCDE, 2002), siendo estos principalmente cuatro: aglomeración, cambios en la producción de mercados imperfectamente competitivos, suministro de mano de obra, y relocalización (Mackie y Worsley, 2013).

Con respecto a la aglomeración y concentración, la literatura indica que una reducción del costo de transporte tiene efectos en la forma en que las empresas se relacionan en un mercado dentro de un territorio. Según Graham (2007) se generan dos fenómenos acumulativos: economías de localización y economías de urbanización. El primer fenómeno, externo a la firma pero interno a la industria, corresponde a los beneficios sobre las firmas de una industria que se ubican relativamente próximas, al existir múltiples proveedores competitivos, desarrollarse mano de obra especializada (división del trabajo) y sinergias en I\&D . El segundo fenómeno, externo a la firma y a la industria pero interno al territorio, corresponde a los beneficios que perciben las empresas por existir un desarrollo urbano, lo que incluye al conocimiento, la cultura, la investigación, y la red de servicios y transporte.

Por otra parte, la concentración aumenta la demanda de viajes y la infraestructura de transporte se vuelve menos eficiente (aumentan los tiempos de viaje, costos, emisiones, etc.). Cuando los efectos de la congestión dominan por sobre las externalidades positivas de la aglomeración, existe una relación entre concentración y baja productividad laboral promedio del territorio (Ciccone, 2002).

Para medir el efecto de economías de aglomeración, la literatura menciona indicadores de productividad media (Vickerman, 2007), densidad del empleo (Ciccone, 2002), y productividad por sector económico (Graham, 2007).

Con respecto a la relación entre transporte y productividad, esta es relativamente simple. Si se consideran los beneficios de la infraestructura afectando a los costos de los factores de producción, los territorios con mejor infraestructura serán más productivos. Esto no considera la forma en que las actividades utilizan la infraestructura en la economía específica, por lo que no sería sorprendente que un territorio cuente con más infraestructura que otro, pero que esta sea inadecuada para las necesidades de dicho territorio y por lo tanto sus beneficios agregados, en productividad, sean comparativamente menores.

Respecto a la mano de obra, una mejora en el transporte aumenta el mercado laboral dado que el tiempo y/o costo de viaje disminuye, por lo que las empresas tienen acceso a un mayor número de trabajadores. Lo anterior es beneficioso pues una mayor competencia en el mercado laboral disminuye los precios y, también, porque se tiene acceso a mano de obra más especializada (uno de los beneficios de la aglomeración). Por otro lado, las mejoras en infraestructura pueden incrementar la competencia por trabajo con mano de obra de fuera del territorio, lo que pudiera resultar perjudicial para la mano de obra local.

Finalmente, si bien teóricamente en el largo pla- 
zo el efecto en la competencia es neutro, es posible encontrar algunos efectos en este ámbito. Dentro de una red en desarrollo (localidades conectadas por caminos dentro de un radio de influencia mutua), existe una fuerte competencia entre sus nodos (localidades), sobre un proceso de valorización-desvalorización en el tiempo (Joignaux y Langumier, 2004).

Como lo indica un reporte de Nueva Zelanda (Kernohan y Rognlien, 2011), a pesar de la relación teórica entre transporte y competitividad de los mercados, la relación real es más bien complicada encontrándose que las mejoras en el transporte pudieran reducir la competitividad en industrias con economías de escala. En Vickerman (2007) se menciona que en algunos casos condiciones desmejoradas de transporte pueden servir como una barrera útil para proteger a una región de competencia más agresiva desarrollada en una región mayor que disfruta de mayores economías a escala en su producción.

Los mayores efectos en la competencia se observarían cuando la mejora en el transporte signifique una disminución importante de los costos tal que restructuraciones de mercado importantes se desarrollen, por ejemplo introduciendo competencia a previos monopolios locales protegidos.

\section{Metodología}

El presente trabajo se enmarca en un desarrollo experimental, de terreno, ex-post, de naturaleza meso-económica, no exhaustiva, específica a cada espacio tratado. Para el estudio de la relación entre la variación en la condición de conectividad y el cambio en el nivel de desarrollo de los territorios, se determinarán las principales dimensiones del desarrollo socio-económico que son posible vincular con mejores condiciones de conectividad.

Por su parte, para el estudio cualitativo de las percepciones que sus habitantes tienen respecto de los beneficios derivados de las iniciativas de conectividad, se utilizarán entrevistas en profundidad, aplicadas en ciertos territorios.

Dado que no se cuenta con indicadores de ingreso y pobreza a nivel de territorios subcomunales, e incluso a nivel comunal, algunos de ellos son estimados utilizando técnicas estadísticas. Se utilizará la comuna como unidad de análisis para este trabajo.

La conectividad se evaluará vinculada a la provisión de infraestructura de transporte, utilizando la inversión pública anual en infraestructura de caminos, carreteras, puentes, túneles, puertos, aero- puertos, aeródromos, entre otros, expresada a nivel comunal, entre el año 1991 y 2012. Mediante un proceso de análisis manual con apoyo de una herramienta SIG y la información que maneja el Sistema Nacional de Inversiones (SNI), se asoció a cada proyecto la o las comunas que forman el área de influencia de un proyecto o IDI. En base a estos datos de inversión, se generaron los percentiles para los periodos de tiempo correspondientes.

Dado que interesa analizar la evolución temporal de las dimensiones de desarrollo, se utiliza para cada unidad territorial un indicador de evolución o variación de la dimensión, en un periodo de tiempo, respecto de la variación promedio a nivel nacional. Estos indicadores se llaman indicadores de cambio (Rimisp, 2013), y están enfocados en la evolución temporal de las brechas territoriales al interior del país en cada dimensión de análisis. El análisis se basa en la observación de estadísticos descriptivos de tendencia central y variación de los indicadores de cambio de las dimensiones de análisis, para cada quintil de inversión pública en conectividad en el quinquenio (o decenio) correspondiente.

Los ámbitos del desarrollo que serán estudiados, sus efectos socio-económicos teóricos, los indicadores asociados y la procedencia de los datos con los que se harán los análisis cuantitativos, se resumen en el cuadro 1.

\section{Diseño de la entrevista}

En una segunda fase, se realiza un análisis comparado entre seis comunas que han mostrado resultados distintos en sus indicadores de desarrollo identificados en la fase cualitativa, para niveles de inversión en infraestructura de conectividad similares.

La elección de las comunas en las cuales realizar las entrevistas, buscó aislar las características que pudieran explicar la apropiación de beneficios derivados de una mejor conectividad, para lo cual se focalizará en comunas que pertenezcan a los últimos quintiles de inversión en infraestructura. Otro rasgo que se consideró es que la inversión se haya concentrado entre los años 2006 y 2010, pues es principalmente en este periodo que los datos disponibles permiten hacer el análisis cuantitativo utilizando indicadores de cambio. Se descartaron aquellas comunas que alojen a la capital regional, pues en virtud de los observado en la experiencia francesa, los efectos inducidos se manifiestan de manera más importante en una región de desarrollo medio que en polos de gran desarrollo (Joignaux y Langumier, 2004). Finalmente, se considera que las comunas sean heterogéneas respecto del porcentaje de 
Cuadro 1: Ámbitos e indicadores seleccionados para análisis

\begin{tabular}{|c|c|c|}
\hline Ámbito & Efecto socio-económico & Indicador relacionado \\
\hline \multirow[t]{2}{*}{ Ingresos individuales } & \multirow{2}{*}{$\begin{array}{l}\text { Incremento de la productividad de la fuerza laboral, al } \\
\text { disminuir el tiempo que las personas ocupan viajando se } \\
\text { dispone de más para actividades productivas, lo que se } \\
\text { asocia al aumento de los ingresos. }\end{array}$} & $\begin{array}{l}\text { Variación del ingreso per capita prome- } \\
\text { dio a nivel comunal. }\end{array}$ \\
\hline & & $\begin{array}{l}\text { Variación del porcentaje de personas en } \\
\text { situación de pobreza monetaria a nivel } \\
\text { comunal. }\end{array}$ \\
\hline \multirow[t]{2}{*}{ Mercado laboral } & $\begin{array}{l}\text { Aumento del mercado laboral, al aumentar las alternativas de em- } \\
\text { pleo en una misma isócrona. }\end{array}$ & Variación de la tasa de ocupados. \\
\hline & $\begin{array}{l}\text { Incremento de la productividad de la fuerza laboral, al disminuir } \\
\text { el tiempo que las personas ocupan viajando se dispone de más } \\
\text { para actividades productivas. }\end{array}$ & $\begin{array}{l}\text { Variación porcentual de la renta media } \\
\text { trabajadores dependientes en el perio- } \\
\text { do. }\end{array}$ \\
\hline \multirow[t]{2}{*}{ Competitividad de mercado } & $\begin{array}{l}\text { Aumento de la posición competitiva derivado de una disminución } \\
\text { de los costos productivos y un acceso a los mercados mejorados. }\end{array}$ & $\begin{array}{l}\text { Variación porcentual promedio de las } \\
\text { ventas de las empresas en el periodo. }\end{array}$ \\
\hline & Exposición a empresas centralizadas más eficientes. & $\begin{array}{l}\text { Variación porcentual promedio del } n^{\circ} \text { de } \\
\text { empresas en el periodo. }\end{array}$ \\
\hline Relaciones comerciales & $\begin{array}{l}\text { Mejora de la relaciones comerciales entre empresas (proveedor- } \\
\text { productor por ejemplo). }\end{array}$ & $\mathrm{S} / \mathrm{I}$ \\
\hline Turismo & $\begin{array}{l}\text { Aumento de la demanda turística pues el "destino" se vuelve más } \\
\text { "accesible" para las personas. }\end{array}$ & $\begin{array}{l}\text { Demanda turística/gasto por turis- } \\
\text { mo/oferta turística a nivel comunal. }\end{array}$ \\
\hline Migración & $\begin{array}{l}\text { Mejoras en la conectividad disminuyen el costo generalizado para } \\
\text { la migración. Personas que antes decidían no migrar por el costo, } \\
\text { ahora pueden cambiar de decisión. }\end{array}$ & $\begin{array}{l}\text { Tasa crecimiento poblacional a nivel co- } \\
\text { munal. }\end{array}$ \\
\hline \multicolumn{3}{|c|}{ Variables de control } \\
\hline Gasto en salud & & Ingreso total per capita en salud \\
\hline Gasto en Educación & & Ingreso per capita educación \\
\hline $\begin{array}{l}\text { Número de organizaciones } \\
\text { comunales }\end{array}$ & & Número de organizaciones comunales \\
\hline
\end{tabular}

población en situación de aislamiento, según metodología Subdere (2013). De las comunas candidatas, se seleccionaron las comunas de Huara, Penco, Lebu, Pica, Camiña y Coronel. En el cuadro a continuación se muestra el porcentaje de la inversión en infraestructura de conectividad total de la comuna que se concentra en el quinquenio 2006-2010, el porcentaje de la población en condición de aislamiento, y los indicadores de desarrollo calculados en la fase cuantitativa.

La cantidad de actores a entrevistar no se decidió por criterios estadísticos, por el contrario, se siguió una estrategia de agregación de conocimiento progresivo. Estas entrevistas semi-estructuradas se aplicaron de forma presencial a actores claves: funcionarios municipales y habitantes de la comuna con énfasis aquellos que sean dirigentes comunales. Se realizaron entre 4 y 5 entrevistas aproximadamente por comuna, cuyo audio fue registrado y luego transcrito. En total se realizaron 28 entrevistas.

Con la ayuda del programa computacional QDA Miner 4 Lite, se procedió a codificar cada entrevista transcrita. Se utilizaron 9 clasificaciones de códigos y 32 códigos, los que se muestran en el cuadro 3.

\section{Resultados}

\section{Resultado del análisis cuantitativo}

Las comunas que presentan valores positivos en todos los indicadores, es decir, que se han desarrollado más que el promedio nacional en estas dimensiones, son solo tres: Arica, Copiapó y Coquimbo (todas capitales regionales). De ellas, sólo Arica pertenece a un quintil de inversión bajo (segundo quintil para los quinquenios 2007-2011). Las otras dos comunas pertenecen a los últimos quintiles de inversión.

Por el contrario, 104 comunas aparecen con todos sus indicadores con valores negativos, es decir, se encuentran en una situación de rezago respecto del promedio nacional. Estas comunas están distribuidas en todos los niveles de inversión en infraestructura, sugiriendo que más inversión no se relaciona con mejor desarrollo de los territorios. De ellas, 26 se encuentran en los dos últimos quintiles de inversión (alta inversión relativa) en los quinquenios analizados. En la cuadro 4 se indican las regiones a las que pertenecen estas comunas.

\section{Inversión en infraestructura y pobreza}

El indicador de cambio de la pobreza muestra que en general las comunas han reducido el número de personas en situación de pobreza en una magnitud mayor, aunque similar, al promedio nacional. So- 
Cuadro 2: Comunas para análisis cualitativo

\begin{tabular}{|c|c|c|c|c|c|c|c|c|c|c|}
\hline \multirow{2}{*}{ Comuna } & \multirow{2}{*}{$\begin{array}{l}\text { Estado de } \\
\text { desarrollo }\end{array}$} & \multirow{2}{*}{$\begin{array}{l}\text { Inversión total } \\
2006-2010[\%]\end{array}$} & \multirow{2}{*}{$\begin{array}{l}\text { Población } \\
\text { aislada [\%] }\end{array}$} & \multicolumn{7}{|c|}{ Indicadores de desarrollo } \\
\hline & & & & Ingreso & Pobreza & Población & $\mathrm{N}^{\circ}$ Empr. & Ventas & $\mathrm{N}^{\circ}$ Trab & Renta Trab \\
\hline Huara & Rezagada & 53,7 & 44 & $-1,16$ & $-0,57$ & $-0,17$ & $-0,32$ & $-0,14$ & $-0,23$ & $-0,18$ \\
\hline Penco & Rezagada & 53,2 & 0 & $-0,31$ & $-1,11$ & 0,06 & $-0,19$ & $-0,12$ & $-0,02$ & $-0,11$ \\
\hline Lebu & Rezagada & 34,9 & 4 & $-0,85$ & $-1,01$ & $-0,18$ & $-0,32$ & $-0,14$ & $-0,24$ & $-0,15$ \\
\hline Pica & Con la media & 51,9 & 16 & 0,07 & 7,88 & 0,27 & $-0,26$ & $-0,14$ & $-0,23$ & $-0,17$ \\
\hline Camiña & Con la media & 51,9 & 22 & $-0,32$ & 7,77 & $-0,21$ & $-0,33$ & $-0,14$ & $-0,24$ & $-0,18$ \\
\hline Coronel & Con la media & 31,7 & 2 & $-0,42$ & $-0,68$ & 0,29 & 0,40 & $-0,05$ & 0,21 & 0,00 \\
\hline
\end{tabular}

Fuente: Elaboración propia

Cuadro 3: Matriz conceptual categorías y códigos para análisis de entrevistas

\begin{tabular}{|c|c|}
\hline Categorías & Códigos \\
\hline \multirow{2}{*}{ Ingresos } & Percepción ingresos promedio \\
\hline & Actividades principales \\
\hline \multirow{4}{*}{ Mercado laboral } & Cantidad empleo \\
\hline & Destinos para trabajar \\
\hline & Calidad empleo \\
\hline & Relación conectividad cantidad/calidad empleo \\
\hline \multirow{4}{*}{ Mercado } & Precios \\
\hline & Variedad \\
\hline & Destinos para comprar/vender \\
\hline & Relación conectividad precios/diversidad \\
\hline \multirow{4}{*}{ Turismo } & Potencial turístico \\
\hline & Oferta turística \\
\hline & Relación conectividad turismo \\
\hline & Evolución demanda \\
\hline \multirow{6}{*}{ Conectividad } & Obras relevantes o mejoras \\
\hline & Evolución conectividad \\
\hline & Impacto en calidad de vida \\
\hline & Inversión \\
\hline & Peajes \\
\hline & Factores evaluación relevantes \\
\hline \multirow{2}{*}{ Migración } & Tendencia migración \\
\hline & Relación conectividad migración \\
\hline \multirow{4}{*}{ Salud } & Calidad salud \\
\hline & Destinos salud \\
\hline & Relación conectividad calidad/cobertura \\
\hline & Cobertura salud \\
\hline \multirow{3}{*}{ Educación } & Cobertura educación \\
\hline & Destinos educación \\
\hline & Relación conectividad cobertura educación \\
\hline \multirow{3}{*}{ Organizaciones sociales } & Numero organizaciones \\
\hline & Capacidad de influir \\
\hline & Interés por organizarse \\
\hline
\end{tabular}

Fuente: Elaboración propia

Cuadro 4: Número de comunas rezagadas con alta inversión en infraestructura por región

\begin{tabular}{|l|c|c|}
\hline Región & $\begin{array}{c}\mathbf{N}^{\circ} \text { comunas rezagadas } \\
\text { con alta inversión }\end{array}$ & $\begin{array}{c}\mathbf{N}^{\circ} \text { comunas } \\
\text { por región }\end{array}$ \\
\hline Región de Tarapacá & 1 & 7 \\
\hline Región de Atacama & 1 & 9 \\
\hline Región de Coquimbo & 1 & 15 \\
\hline Región de Valparaíso & 6 & 38 \\
\hline Región del Biobío & 3 & 54 \\
\hline Región de La Araucanía & 2 & 32 \\
\hline Región de Los Lagos & 4 & 30 \\
\hline $\begin{array}{l}\text { Región Metropolitana de } \\
\text { Santiago }\end{array}$ & 8 & 52 \\
\hline Total & 26 & 237 \\
\hline
\end{tabular}

Fuente: Elaboración propia 
bresalen, sin embargo, algunas comunas del cuarto quintil de inversión (Putre, Pozo Almonte, Camiña y Pica) que redujeron su tasa de pobreza en una proporción muy superior al resto de las comunas analizadas.

El cuadro 5 muestra los estadísticos relacionados con este indicador para cada nivel (quintil) de inversión en infraestructura de conectividad.

Llama la atención que de aquellas comunas en las que más se invirtió en infraestructura (pertenecientes al último quintil de inversión), más de la mitad mostraron un cambio en el indicador de pobreza negativo, es decir que el número de personas en situación de pobreza (tasa de pobreza) en dichas comunas disminuyó menos que lo observado en promedio a nivel nacional, o incluso aumentó.

Es más, para cada nivel de inversión (cada quintil), más del $25 \%$ de las comunas disminuyó su nivel de pobreza en una proporción menor al promedio nacional (o incluso aumentó su proporción de pobreza). Lo anterior indica que la evolución en los niveles de pobreza comunal, comparados con el resultado nacional, no dependería del monto de las inversiones en conectividad que se realizan.

\section{Inversión en infraestructura y mercado labo- ral}

El indicador de cambio del número de trabajadores dependientes muestra en general que las comunas se comportaron por debajo del promedio nacional $^{2}$, en el quinquenio analizado. En efecto, se observa que más del $75 \%$ de las comunas para cada nivel de inversión en conectividad muestra este comportamiento de variación inferior al promedio nacional.

Sin embargo, se observa que para mayores niveles de inversión aumenta la brecha entre las comunas (los valores máximos aumentan considerablemente mientras los mínimos permanecen relativamente constantes).

Por su parte el indicador de cambio de la renta neta de los trabajadores dependientes muestra una variación que en general es menor que el comportamiento promedio nacional en el quinquenio. Así, en más del $75 \%$ de las comunas analizadas la renta disminuyó o aumentó menos que la variación promedio nacional ${ }^{3}$.

\footnotetext{
${ }^{2} \mathrm{El}$ número de trabajadores dependientes a nivel comunal aumentó en promedio 6.561 personas entre los años 2007 y 2011.

${ }^{3} \mathrm{El}$ aumento promedio de la renta neta de los trabajadores (suma total) entre los años 2007 y 2011 fue de \$1.494.056.
}

Se observa, al igual que con otros indicadores, un aumento de los valores máximo del indicador a medida que el nivel de inversión en infraestructura aumenta, es decir un aumento en la desigualdad en la dimensión, sin embargo las variaciones están por debajo de una variación estándar en la mayoría de las comunas.

\section{Inversión en infraestructura y competitivi- dad}

El análisis muestra que en más del $50 \%$ de las comunas el número de empresas aumentó en menor número (o disminuyó) respecto al promedio nacional en el quinquenio analizado.

Se observa, por otro lado, que al aumentar la cantidad de recursos que se ha invertido en las comunas aumenta también la dispersión de los valores del indicador de cambio asociado. Esto es, los valores máximos aumentan y los mínimos disminuyen, al aumentar la inversión.

Por otro lado, en general las empresas muestran niveles de ventas que variaron en el quinquenio en menor magnitud que la variación media nacional, para los cuatro primeros quintiles, exceptuando las comunas del último quintil de inversión, las que muestran un variación de las ventas mayores en comparación al comportamiento promedio nacional. Sin embargo, sin importar el nivel de inversión en infraestructura, más del $50 \%$ de las comunas vieron una variación de las ventas netas menor al promedio nacional o disminuyeron sus ventas. Incluso, más del $90 \%$ de las comunas pertenecientes a los cuatro primero quintiles de inversión, disminuyeron sus ventas o no superaron la variación media nacional ${ }^{4}$.

\section{Inversión en infraestructura y población}

En general las comunas analizadas muestran que su población aumentó en una menor proporción al del promedio nacional pero con una alta dispersión. Tanto es así que el indicador de cambio de población es negativo para más del $50 \%$ de las comunas en cada quintil de inversión en infraestructura. Es más, en los cuatro primeros quintiles de inversión, más del $75 \%$ de las comunas tienen su indicador menor a cero.

En esta misma línea, llama la atención la relación que existe entre la condición de rezago de las comunas y la variación de su población en el último

\footnotetext{
${ }^{4}$ En promedio las ventas netas de las empresas de las comunas chilenas aumentaron en $\$ 19.301 .087$ entre el año 2007 y 2011.
} 
Cuadro 5: Estadísticos del indicador de cambio de pobreza para cada nivel de inversión

\begin{tabular}{|c|c|c|c|c|c|c|}
\hline & \multicolumn{5}{|c|}{ Quintil según inversión para el decenio 2002 - 2011} \\
\hline & & 1 & 2 & 3 & 4 & 5 \\
\hline \multirow{4}{*}{ Percentiles } & 25 & $-0,24$ & $-0,452$ & $-0,422$ & $-0,469$ & $-0,631$ \\
\hline & 50 & 0,121 & 0,05 & 0,052 & 0,19 & $-0,217$ \\
\hline & 75 & 0,459 & 0,411 & 0,496 & 0,699 & 0,224 \\
\hline & 90 & 0,743 & 0,958 & 1,172 & 1,667 & 0,812 \\
\hline
\end{tabular}

Cuadro 6: Estadísticos del indicador de cambio del número de trabajadores dependientes, según inversión

\begin{tabular}{|c|c|c|c|c|c|c|}
\hline & \multicolumn{6}{|c|}{ Quintil según inversión para el quinquenio 2007 - 2011} \\
\hline & & 1 & 2 & 3 & 4 & 5 \\
\hline \multirow{4}{*}{ Percentiles } & 25 & $-0,232$ & $-0,236$ & $-0,235$ & $-0,234$ & $-0,23$ \\
\hline & 50 & $-0,213$ & $-0,228$ & $-0,222$ & $-0,218$ & $-0,185$ \\
\hline & 75 & $-0,18$ & $-0,199$ & $-0,18$ & $-0,19$ & $-0,025$ \\
\hline & 90 & $-0,097$ & $-0,098$ & $-0,133$ & 0,003 & 0,412 \\
\hline Mínimo & & $-0,265$ & $-0,259$ & $-0,257$ & $-0,603$ & $-0,32$ \\
\hline Máximo & & 0,246 & 0,157 & 0,497 & 1,258 & 1,175 \\
\hline
\end{tabular}

Cuadro 7: Estadísticos del indicador de cambio de la renta neta de los trabajadores dependientes, según inversión

\begin{tabular}{|c|c|c|c|c|c|c|}
\hline & \multicolumn{6}{|c|}{ Quintil según inversión para el quinquenio 2007 - 2011} \\
\hline & & 1 & 2 & 3 & 4 & 5 \\
\hline \multirow{4}{*}{ Percentiles } & 25 & $-0,175$ & $-0,177$ & $-0,176$ & $-0,174$ & $-0,171$ \\
\hline & 50 & $-0,171$ & $-0,173$ & $-0,172$ & $-0,167$ & $-0,139$ \\
\hline & 75 & $-0,153$ & $-0,165$ & $-0,159$ & $-0,152$ & $-0,055$ \\
\hline & 90 & $-0,122$ & $-0,134$ & $-0,12$ & $-0,073$ & 0,312 \\
\hline Mínimo & & $-0,19$ & $-0,189$ & $-0,18$ & $-0,181$ & $-0,181$ \\
\hline Máximo & & 0,051 & 0,068 & 0,288 & 0,75 & 1,047 \\
\hline
\end{tabular}

Cuadro 8: Estadísticos del indicador de cambio en el número de empresas para cada nivel de inversión

\begin{tabular}{llccccc}
\hline \hline & \multicolumn{5}{c}{ Quintil según inversión para el quinquenio $2007-2011$} \\
& & 1 & 2 & 3 & 4 & 5 \\
\hline \multirow{4}{*}{ Percentiles } & 25 & $-0,304$ & $-0,328$ & $-0,327$ & $-0,314$ & $-0,276$ \\
& 50 & $-0,253$ & $-0,282$ & $-0,289$ & $-0,25$ & $-0,145$ \\
& 75 & $-0,183$ & $-0,2$ & $-0,246$ & $-0,155$ & 0,211 \\
Mínimo & 90 & $-0,049$ & 0,058 & 0,003 & 0,404 & 0,91 \\
Máximo & $-0,356$ & $-0,388$ & $-0,419$ & $-1,154$ & $-0,43$ \\
\hline \multicolumn{2}{l}{ Fuente: Elaboración propia en } & 0,444 & 1,538 & 1,235 & 1,38 & 2,466 \\
\hline
\end{tabular}

Cuadro 9: Estadísticos del indicador de cambio de las ventas, según inversión

\begin{tabular}{ccccccc}
\hline \hline & \multicolumn{5}{c}{ Quintil según inversión para el quinquenio $2007-2011$} \\
& & 1 & 2 & 3 & 4 & 5 \\
\hline \multirow{4}{*}{ Percentiles } & 25 & $-0,139$ & $-0,14$ & $-0,14$ & $-0,139$ & $-0,138$ \\
& 50 & $-0,137$ & $-0,138$ & $-0,139$ & $-0,135$ & $-0,12$ \\
& 75 & $-0,129$ & $-0,129$ & $-0,131$ & $-0,126$ & 0,02 \\
& 90 & $-0,107$ & $-0,1$ & $-0,11$ & $-0,068$ & 0,271 \\
\hline \hline
\end{tabular}

Fuente: Elaboración propia en base a los datos analizados 
Cuadro 10: Estadísticos del indicador de cambio del número de habitantes para cada nivel de inversión

\begin{tabular}{lcccccc}
\hline \hline & & Quintil según inversión para el decenio $2002-2011$ \\
& & 1 & 2 & 3 & 4 & 5 \\
\hline \multirow{4}{*}{ Percentiles } & 25 & $-0,209$ & $-0,201$ & $-0,204$ & $-0,209$ & $-0,205$ \\
& 50 & $-0,179$ & $-0,172$ & $-0,188$ & $-0,167$ & $-0,127$ \\
& 75 & $-0,138$ & $-0,139$ & $-0,047$ & $-0,098$ & 0,172 \\
Mínimo & 90 & 0,058 & $-0,049$ & 0,219 & 0,036 & 1,095 \\
Máximo & $-0,362$ & $-0,263$ & $-0,333$ & $-0,46$ & $-2,765$ \\
\hline \multicolumn{7}{l}{ Fuente: Elaboración propia en } \\
\end{tabular}

decenio. La población en promedio aumenta mucho más en aquellas comunas adelantadas, comparado con aquellas comunas rezagadas. Las dinámicas migratorias de las localidades es un factor relevante que debe ser analizado en mayor detención.

\section{Inversión en infraestructura y brecha inter- comunal}

Se considera ahora, para cada indicador de cambio, la distancia entre el punto de corte del percentil 50 (50\% de las comunas está por debajo de este valor) y del percentil 90 (el $10 \%$ de las comunas está por encima de dicho valor), lo que se llamará brecha intercomunal y se puede apreciar en el cuadro 11.

Se observa que dicha distancia tiende a aumentar a medida que aumenta el nivel de inversión en infraestructura. En particular, para los indicadores de venta de las empresas, renta de los trabajadores y número de trabajadores dependientes, prácticamente no se observa cambio en la brecha a medida que aumenta la inversión en infraestructura, salvo para el último quintil de inversión, aumentando la distancia entre las comunas que lo hacen mejor de las otras. Lo anterior se puede apreciar mejor en la figura 1. Pudiera interpretarse este fenómeno como que el aumento en la inversión genera condiciones para que sólo algunas comunas se desarrollen más o mejor y no genera el mismo cambio en todas, lo que deberá analizarse con mayor atención.

\section{Resultado del análisis cualitativo}

\section{Empleo (mercado laboral) e ingresos}

La percepción prácticamente unánime es que la cantidad de empleo que se ofrece en estas comunas es insuficiente para la cantidad de personas que quieren trabajar. Sin embargo, prácticamente todos los entrevistados indican que la oferta de empleo ha venido aumentando en los últimos años. Los mati- ces a esto aparecen al considerar personas que están en edad de trabajar pero no quieren (se mencionan factores culturales/familiares) o no pueden (minusvalía).

En relación a los sueldos, la gran mayoría menciona que son bajos, fuertemente complementados con aportes estatales (subsidios y otras asistencias).

Se observan dos tipos de comunas, aquellas donde pareciera que es habitual que se viaje por motivos de trabajo y otras que no (Camiña y Coronel). También se observan comunas cuya actividad económica principal pareciera ser agrícola, y otras donde se aprecia un mayor dinamismo (las comunas del Biobío muestran un dinamismo económico aparente mayor que las de Tarapacá).

Se menciona reiteradamente la migración de personas a otros sectores del país (capital regional o sectores mineros) en búsqueda de un mejor desarrollo laboral. Se menciona bastante también la llegada de trabajadores de otras comunas e incluso de otros países (en las comunas del norte del país) para satisfacer una demanda por trabajo de baja remuneración. Respecto de los extranjeros, estos estarían trabajando en condiciones laborales cuestionables y a un costo muy inferior al que aspirarían los trabajadores nacionales. En general, los empleos ofrecidos en las comunas serían de baja complejidad y realizados por personas con baja capacitación. Personas con mayor capacitación parecieran migrar a otras ciudades.

En la mayoría de las comunas el Municipio es una fuente de trabajo importante y que ofrece remuneraciones comparativamente altas. Indirectamente también aporta la municipalidad a través de programas pro empleo (pero estos son con salarios menores).

Aparecen también los proyectos de construcción como importantes fuentes de empleo local y temporal. Sin embargo, al parecer muchas veces las empresas constructoras traen destacamento de trabajadores de otros sectores, principalmente para aquellas funciones de mayor valor agregado. 
Cuadro 11: Brecha intercomunal (distancia entre corte percentiles 90 y 50)

\begin{tabular}{lccccc}
\hline \hline \multirow{2}{*}{ Indicador } & Quintil según inversión & \\
& 1 & 2 & 3 & 4 & 5 \\
\hline Cambio en el nivel de pobreza & 0,622 & 0,908 & 1,12 & 1,477 & 1,029 \\
Cambio en el número de trabajadores & 0,116 & 0,13 & 0,089 & 0,221 & 0,597 \\
Cambio en la renta de los trabajadores & 0,049 & 0,039 & 0,052 & 0,094 & 0,451 \\
Cambio en el número de empresas & 0,204 & 0,34 & 0,292 & 0,654 & 1,055 \\
Cambio en las ventas de las empresas & 0,03 & 0,038 & 0,029 & 0,067 & 0,391 \\
\hline \hline
\end{tabular}

Fuente: Elaboración propia en base a datos analizados

Figura 1: Brecha intercomunal para cada quintil de inversión en infraestructura

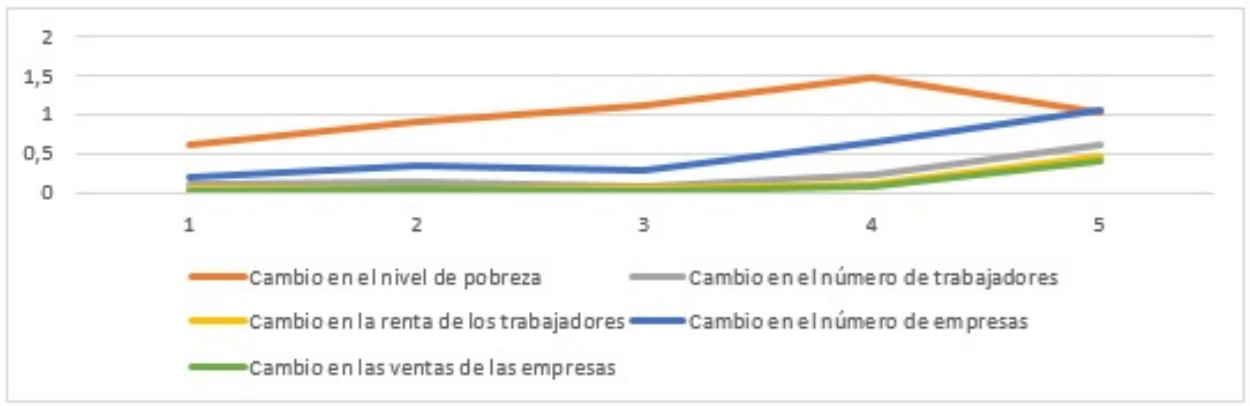

Fuente: Elaboración propia en base a datos analizados

En general los entrevistados perciben una relación entre la conectividad y la posibilidad de acceder a más empleo y/o mejor calidad. La principal relación, estaría dada por el tiempo y costo de viaje, ya que parecieran considerarse dichos factores constituyentes de la calidad del empleo y, por lo tanto, factores a evaluar. Se menciona también que una disminución del tiempo de viaje aumentaría la cantidad de empleo disponible, al aumentar el espacio geográfico potencial para buscar trabajo, mejorando la competencia en el mercado laboral (más de una comuna ofrecen empleo a una misma demanda).

Cabe resaltar que en muchos casos se mencionó el alto costo del transporte público y/o peajes, que hacen disminuir los efectos positivos de las mejoras en infraestructura.

\section{Mercado}

La percepción generalizada es que en estas comunas el precio de los productos es más alto comparado con la principal referencia (capital regional habitualmente), y que en cuanto la variedad de productos esta es menor. Se observaron excepciones en Coronel y Penco donde algunos opinan que el precio es comparable, del mismo modo algunos entrevistados de Pica indicaron que en términos de variedad de productos no se encontraban "tan mal". En el caso particular de Coronel, se menciona que la cercanía con la feria de Lota y el Mercado de Abastos de Concepción es una ventaja para contar con bue- nos precios y variedad de productos (alimentarios al menos).

Otra componente indicada por un entrevistado que justificaría el alza de los precios es la disminución de la producción agrícola, lo que puede deberse al cambio productivo de la tierra (de la agricultura a la silvicultura) o al cambio de la actividad productiva (de actividades primarias a secundarias o terciarias).

En general, los principales factores declarados como causales de la diferencia de precios y variedad fueron el costo del flete, la menor demanda y cierto poder monopólico que tendrían los comercios locales. Sobre el costo de flete se indicó en algunas oportunidades que dicho costo no se vería afectado por mejoras en conectividad, que la tarifa depende mucho más de la distancia (asumiendo que las mejoras en conectividad ya no influirán de forma importante en las distancias recorridas). Respecto a la menor demanda que enfrentan los comercios locales (comparados con aquellos de las ciudades más grandes), esta se ve disminuida porque han incorporado el hábito de viajar y autoabastecerse en las ciudades, disminuyendo la necesidad de consumir localmente (alto nivel de gasto en ciudades). Finalmente, el comercio local pareciera enfocarse a entregar productos perecederos (que no conviene viajar para comprarlos) y de alta demanda (por ejemplo pan) o bien de nicho muy particulares (agujas, hilo), ello sumado a que el principal mercado comercial se encuentra relativamente alejado, pareciera otorgarle a los comercio locales cierto poder monopólico (en caso de necesitar margarina o una aguja para 
cocer no resulta sensato viajar a la ciudad) y por lo tanto estarían cobrando un precio muy elevado. Si eso fuera así, aumentaría el beneficio percibido por abastecerse fuera de la localidad, debilitando aun más el comercio local.

Un cambio relevante en los mercados que se estaría dando en las comunas del Biobío es la aparición de grandes tiendas de venta al detalle (retail), lo que estaría provocando, según algunos entrevistados, la desaparición o al menos reordenamiento de los pequeños comerciantes.

Una dinámica de los mercados que se relaciona con la conectividad tiene que ver con la dependencia que desarrollan las localidades respecto de las ciudades más cercanas (habitualmente la capital regional). Así una localidad que enfrenta un costo y tiempo de viaje, respecto de la ciudad, relativamente mayor al de otra localidad, se desarrollaría con mejores capacidades dado que depende (obligadamente) menos que la otra localidad, de dicha ciudad. Lo anterior no significa que en términos de ingresos o producción económica ambos territorios se comporten necesariamente de una forma particular, pero sí es relevante considerar si este efecto de "autonomía" del centro económico es un factor relevante para comprender el camino de desarrollo que las localidades atraviesan.

Se aprecia una fuerte relación entre el autoabastecimiento y la necesidad de viajar a las capitales por salud, servicios, trámites o, en algunos casos puntuales para vender la producción agrícola. Así, según declaraciones de entrevistados, se "aprovecha mejor el viaje" si regresan con productos para el consumo personal.

Respecto a la conectividad, en general se percibe una relación entre esta y el costo de flete. Sin embargo, la mayoría de las respuestas relevan la disminución del tiempo de viaje de las personas, lo que facilitaría la compra de mejores o más productos (se menciona incluso la posibilidad de cotizar más). Esto último pareciera reforzar la idea de que el hábito de autoabastecerse en las ciudades está bastante instalado.

Cabe destacar que algunas personas indican que las mejoras en conectividad no reducirían los costos de transporte, lo que se debería a que las distancias recorridas no se alterarían. Otro factor que algunas personas consideran relevante es el peaje cobrado por circular en rutas concesionadas, el que sería muy elevado y el resultado final es que no permitiría disminuir el costo del flete a pesar de la mejora de las carreteras.

Finalmente, se resaltó la idea de que la conectividad no influye en nada si no hay dinamismo económico en la localidad, que se pueden tener excelentes carreteras pero no afectan en nada si no hay empleo y sueldos adecuados.

\section{Turismo}

La opinión prácticamente unánime es que estas comunas tienen potencial turístico, que no se ha explotado y que la oferta existente no es suficiente. La excepción sería Pica, en dónde un par de personas indican que existe sobre oferta de alojamiento (principalmente por el aumento en la demanda por alojamiento para los familiares de los trabajadores mineros que gozaban de altos ingresos por lo que preferían traer a sus familiares que viajar de regreso a sus hogares). En el otro extremo se encuentran Penco y Coronel que no tienen oferta de alojamiento, dependiendo completamente de la oferta que provee Concepción.

Es habitual la opinión respecto de la necesidad de contar con "rutas turísticas" y por ende la necesidad de coordinación con otros municipios. Lo que aparentemente no ocurre. Dichas rutas permitirían visibilizar las localidades y sus atractivos. En este sentido, en algunas ocasiones se mencionó que las mejoras viales "ocultaban" las localidades, por ejemplo al construir bypass, dado que las personas ya no transitaban por ellas.

La opinión mayoritaria es que la conectividad forma parte del atractivo que tiene una zona para ser visitada, indicando que si cuesta mucho llegar, los potenciales turistas preferirían no ir. Se resaltó también, la importancia de que los caminos no contaminaran visualmente el paisaje, sobre todo en aquellos lugares en que el potencial turístico está relacionado con la naturaleza, la cultura, la historia, etc. Vale la pena destacar la variable étnica, mencionada en varias ocasiones en las comunas del norte, la importancia de generar un turismo adaptado a la identidad de las personas que habitan allí (descendientes de aimara y quechua), y la necesidad de rescatar "el relato histórico" de los habitantes más antiguos.

Vale la pena mencionar el caso particular de Penco, donde hasta hace algunas décadas atrás operaba un tren de pasajeros. Los entrevistados de esa comuna indican que la eliminación de ese servicio de pasajeros significó una disminución muy importante de turistas a Penco que iban principalmente a la playa.

Rev. Est. de Políticas Públicas, 2016, vol. 3 (junio) 


\section{Conectividad}

En general la percepción de las personas es que la conectividad (entendida como la cantidad y calidad de la infraestructura vial) es buena y que ha venido mejorando, destacando brechas en sectores rurales o territorios con poca población. En este sentido, casi todas las personas opinan que la inversión en conectividad es suficiente. Sin embargo, varios entrevistados indican que las mejoras tardan mucho.

La mayoría opina que el desarrollo de la conectividad tiene un impacto relevante en la calidad de vida de las personas, expresado en diversas dimensiones, pero fundamentalmente en disminución de tiempo, costos y aumento de la seguridad.

Algunos entrevistados destacaron la capacidad de (in)visibilizar un territorio que tiene la conectividad, al (desviar) conducir los viajes por un territorio determinado. Por ejemplo, en Penco se mencionó que el bypass hizo que muchos viajes que se dirigen al sur ya no pasan por el pueblo y por lo tanto se pierde la potencial actividad económica que podría estar asociada al viaje (alimentación, asistencia mecánica, etc.).

Quizás un ejemplo de mayor magnitud es el caso de la ruta $15-\mathrm{CH}$ que conecta Huara y el paso fronterizo en Colchane, el que pasa relativamente alejado de los poblados que se encuentran en los valles y quebradas (incluido el pueblo de Tarapacá, capital de la provincia de Tarapacá peruana entre los años 1878 y 1883). Dicha ruta tiene una vocación claramente productiva, con un alto flujo de camiones entre Bolivia y la Zofri y de buses de pasajeros, y para visitar las localidades cercanas se tienen accesos en malas condiciones y de geometría trabada para la circulación de algunos vehículos.

Es relevante destacar también, que en las comunas del Biobío las rutas enfrentan mucha congestión, por lo que los beneficios que derivarían de las mejoras en infraestructura se diluyen. Por lo tanto, aparece en las opiniones el tema del tráfico y la importancia que ello tiene para la calidad de vida.

Respecto de las brechas en conectividad se mencionó reiteradamente la relativa baja conectividad con regiones vecinas o incluso con comunas vecinas si estas no estaban en el camino hacia la capital regional. La red vial se configura fuertemente en una estructura radial, fomentando la dependencia con el centro económico regional.

\section{Migración}

Lo primero es hacer notar que en ambas regiones ocurrieron desastres naturales hace pocos años atrás: terremoto y tsunami en Biobío el año 2010 y terremoto en Tarapacá el año 2014 (se agrega un aluvión en el valle de Camiña el 2012).

Se observa una marcada diferencia entre las comunas, generándose dos grupos: aquellas en que se percibe un aumento de la población total y aquellas en las que por el contrario pareciera que la población disminuye. Las comunas del primer grupo son Pica, Penco y Coronel.

De todas maneras en todas las comunas se declara que muchas personas migran en búsqueda de mejores condiciones laborales y de capacitación (estudio). Es prácticamente unánime la opinión de que la población joven emigra, y en caso que lleguen, se trataría principalmente de adultos terminando sus años activos o adultos mayores, o de personas provenientes de localidades cercanas que estarían en condiciones menos ventajosas (como por ejemplo a Coronel estaría llegando muchas personas de Lota). Por otro lado, el aumento de la población en Coronel y Penco se explicaría por la migración importante, desde otras localidades cercanas, de personas atraídas por la disponibilidad de terrenos (y de proyectos habitacionales, principalmente viviendas sociales) y la cercanía con Concepción.

De las cuatro comunas cuya población estaría envejeciendo (Pica, Lebu, Camiña y Huara), solo la comuna de Pica parece ser atractiva para los adultos mayores de la región, según cuentan los entrevistados. Por lo tanto además de la emigración de jóvenes, se suma la llegada de nueva población mayor a cincuenta años.

Un par de entrevistados mencionan el proceso migratorio como "escalonado", es decir las personas de localidades rurales y de bajo desarrollo, pasan a vivir a la cabecera comunal, al mismo tiempo algunas personas de la cabecera comunal buscan mejores oportunidades en comunas aledañas que ofrecen mejores condiciones (Pozo Almonte o Alto Hospicio en el caso de la región de Tarapacá, Coronel o Cañete en el caso del Biobío). A su vez, los habitantes de dichas comunas relativamente más aventajadas estarían buscando mejores oportunidades en las capitales regionales cercanas (se menciona Arica, Iquique, Temuco y Concepción), y finalmente aparece la capital nacional como aspiración.

Es importante señalar también que se mencionó en algunas entrevistas el factor de identidad con el territorio, y como ello diferencia a quienes habitan hace tiempo en él de los que vienen llegando. Sin 
constituir una opinión clara de los entrevistados, la identidad y el arraigo podrían ser factores relevantes a la hora de analizar profundamente las causas del desarrollo territorial.

Respecto a la relación entre conectividad y migración, las respuestas son mayoritarias en indicar que la relación está dada más bien en la elección del destino a migrar (una característica más a evaluar respecto de los potenciales destinos) y no en la decisión de salir de un lugar (los factores claves para esta decisión estarían relacionados principalmente con oportunidades y desarrollo laboral y expectativas de mejor educación). Sin embargo, y bajo el mismo argumento, la decisión de salir de un territorio pudiera verse influenciada por las mejoras en la conectividad, principalmente si estas son importantes. Esto último fue declarado por una minoría de los entrevistados.

\section{Conclusiones}

La principal conclusión de la presente investigación es que se aprecia una baja relación entre las dimensiones de desarrollo socio-económico consideradas y el nivel de inversión en infraestructura de transporte, es decir que los resultados comunales observados en general es similar sin importar si se invierte más o menos.

En particular, se observa que la inversión en infraestructura poco influye en generar condiciones homogéneas para el desarrollo del mercado laboral y competitividad de las empresas, por el contrario, el aumento en la inversión pública en conectividad pareciera generar condiciones para que sólo algunas comunas se desarrollen de manera significativamente mejor, mientras la mayoría queda en condiciones intermedias o incluso de deterioro.

Lo anterior es una manifestación de un problema en la política pública de inversión en infraestructura de transporte, ya sea en la definición de sus objetivos o en su implementación.

Claramente, con esto no se quiere hacer creer que la conectividad y su mejora continua son irrelevantes para las condiciones de desarrollo. No se intenta cuestionar lo relevante de disminuir los costos de transporte mejorando la calidad de vida de las personas y la productividad de los principales sectores económicos. Tampoco se pone en duda el impacto que tiene para los habitantes de las localidades poder acceder en menor tiempo y costo a los servicios públicos. De hecho, es interesante notar que la percepción mayoritaria de las personas es que existe una relación positiva entre mejoras en la conectivi- dad y las dimensiones de desarrollo estudiadas. Sin embargo, al profundizar en el análisis, la opinión general es que el nivel de desarrollo es bajo. Es decir, la inversión en infraestructura de conectividad no ha logrado cambiar la sensación de rezago que tienen las personas entrevistadas, no logra cambiar la calidad de vida de las personas de manera que sea valorada.

Las posibles causas de ello, en virtud de lo estudiado, son cuatro y están interrelacionadas:

a) El objetivo de la política de infraestructura no ha sido el desarrollo de los territorios.

La red de transporte se ha configurado desde los centros de mayor dinamismo hacia la periferia, en una estructura que podríamos llamar radial. Así, es habitual que los caminos estén pavimentados entre la capital regional y la cabecera comunal pero no desde esta última hacia localidades más alejadas. Peor aún, la conectividad entre comunas de distintas regiones es bastante limitada (por ejemplo entre Pica y Ollague, o entre Lebu y Temuco). El desarrollo de las localidades pareciera siempre mirar hacia la capital regional, el polo de mayor desarrollo, dándole la espalda a las localidades vecinas o más pequeñas en particular si pertenecen a otra administración. La falta de conectividad local restringe las relaciones funcionales que se pudiesen dar entre dichos territorios y se estaría perdiendo entonces una oportunidad de desarrollo (Berdegué y Proctor, 2014) local. En general las obras de infraestructura no tienen incorporada la visión y necesidades de los territorios por donde pasa.

b) Los beneficios de la infraestructura no están debidamente identificados.

Algunas obras de infraestructura tienen una vocación productiva, orientadas para el transporte de grandes volúmenes de carga y gran cantidad de pasajeros (rutas internacionales, puertos, aeropuertos, pasos fronterizos, entre otros) por lo que deben permitir velocidades relativamente altas, con niveles de seguridad adecuados para el correcto transporte de bienes y personas. Se orientan a ser un viaducto sobre los territorios, puesto que pasar por ellos significaría un deterioro en su estándar. Así, tienen un efecto acotado o incluso negativo sobre los territorios intermedios. El proceso de preinversión y ejecución de obras de infraestructura usado actualmente no identifica claramente los beneficios socio-económicos (en particular los "amplios"), ni mediante cuáles mecanismo dichos beneficios podrían generarse y ser apropiados por la comunidad.

c) No existe suficiente investigación sobre las rela- 
ciones de estos ámbitos.

A pesar de que las iniciativas de inversión deben justificarse socialmente utilizando herramientas económicas, no existe conocimiento respecto de la real relación entre inversión en infraestructura y desarrollo territorial, por lo tanto los efectos locales resultantes son meras casualidades y no planificados. Hoy no se estudian las interacciones múltiples y diversas entre una nueva dotación de infraestructura y una economía territorial, incluyendo los actores susceptibles de adoptar políticas locales de desarrollo, para mejorar aún más y aprovechar dicha dotación. A $\mathrm{su}$ vez no existen estudios expos que verifiquen o refuten las hipótesis que se plantearon al momento de la evaluación de un proyecto, ni menos la orientación estratégica de una política pública.

\section{Recomendaciones}

\section{Proyectos con participación y flexibi- lización presupuestaria}

Las obras de conectividad deben elaborarse con real participación de los actores que serían sus beneficiarios, considerando y respondiendo a cada consideración de la ciudadanía.

Para asegurar que se pueda dar respuesta adecuada (lo que no significa de modo alguno acoger cada demanda) a las aprensiones y comentarios, puede ser necesario muchas veces realizar análisis no considerados en las etapas previas de formulación de proyecto (impacto en la actividad económica, efecto en la calidad urbanística, etc.). Por lo tanto se requiere permitir que los proyectos modifiquen la disponibilidad de recursos disponible en un periodo dado, cuando se pueda justificar que las dudas emanadas por parte de la ciudadanía no fueron relevadas con anterioridad y deben ser respondidas rápidamente.

Del mismo modo, se propone flexibilidad presupuestaria para permitir realizar mitigaciones no consideradas en etapas anteriores de diseño, en casos debidamente justificados. Para ello podría existir una glosa presupuestaria que represente un porcentaje del presupuesto de cada proyecto para ser utilizado para estos fines.

\section{Tratar los proyectos de conectividad según su vocación}

Resulta fundamental que los proyectos de conectividad sean caracterizados según sus funcionalidades primordiales y a quienes beneficiarían, y estas sean transparentemente difundidas. De este modo las personas no podrían esperar que un camino principalmente productivo industrial ofrezca las condiciones necesarias para desarrollar otras dimensiones (por ejemplo no sería posible pensar en turismo étnico si por el camino circulará una gran cantidad de camiones), y no se debería criticar los resultados si las industrias agrícolas de la zona han mejorado su competitividad.

Es necesario que se precise, en virtud de la vocación de la infraestructura, a quienes está dirigido el desarrollo que se le imputa a la obra y mediante cuales mecanismos llegarán a las personas.

Así, los proyectos con vocaciones distintas no deberían competir por recursos, al menos no bajo las mismas reglas: una carretera industrial cuyos beneficiarios son empresarios exportadores de materias primas, no debería compararse con una carretera que conecta los poblados que pertenecen a una misma etnia y comparten ritos religiosos. Por ejemplo, los proyectos con vocación intrínsecamente social deberían evaluarse con enfoque costo-eficiencia y ser financiados con recursos especiales para este tipo de proyectos, mientras que aquellos proyectos industriales podrían evaluarse mediante costobeneficio y entrar al sistema de concesiones públicas.

\section{Mejora en el registro de datos del SNI}

Parte fundamental del presente análisis se basó en la información sobre proyectos (Iniciativas de Inversión) que maneja el Sistema Nacional de Inversiones (SNI), sin embargo la componente territorial está débilmente desarrollada. Lo anterior repercute en que análisis de inversión a nivel comunal sean prácticamente imposibles de realizar sin invertir en recursos importantes.

Por otro lado la información respecto de la inversión efectivamente ejecutada es compleja de obtener y no siempre existe información respecto de la ejecución de los proyectos.

Se propone revisar el SNI para fortalecer al componente territorial y de ejecución de las Iniciativas de Inversión, con foco en el registro administrativo de la inversión realizada por cada sector a distintos niveles territoriales. 


\section{Desarrollar conocimiento sistemático sobre el equipamiento de transporte}

Para replicar y sistematizar el esfuerzo realizado en este trabajo de investigación, se propone establecer las condiciones para realizar las mediciones necesarias, en el lugar y momento adecuados, con el objeto no solamente de evaluar o estimar de la mejor forma posible los impactos positivos y negativos que distintas obras de conectividad tienen sobre los territorios, sino además, y como lo indica (Langumier, 2002) "las interacciones múltiples y diversas entre una nueva dotación (de infraestructura) y una economía territorial que incluye sus propios actores susceptibles de adoptar políticas locales de desarrollo, para mejorar aún más y aprovechar la infraestructura (...) dentro de un contexto dado".

Es elaborar conocimiento para aportar con elementos científicamente construidos a la pregunta: ¿la infraestructura contribuye a la creación de excedentes económicos en los territorios servidos por ella? Y en cuáles condiciones, o cuáles formas, o para cuáles agentes económicos sí y cuáles no, y cómo estos se apropian de los beneficios (Joignaux y Langumier, 2004).

Estas estructuras o sistemas de medición pueden incluirse en las condiciones de concesión de operación de grandes proyectos o desarrollarse a nivel territorial con fondos públicos y/o privados.

Se recomienda la experiencia francesa, en particular los aprendizajes reportados por (Langumier, 2002 ) sobre los tres observatorios desarrollados posteriores a 1985. Destaca la recomendación sobre la necesaria participación de actores universitarios y de investigación en conjunto con la de tomadores de decisión y la comunidad, y principalmente el periodo de observación el cuál debería incluir la fase anterior a la obra, la obra y después de la puesta en servicio, permitiendo así tener una línea base con la cual compara la evolución.

\section{Referencias}

Barca, F., McCann, P., y Rodríguez-Pose, A. (2011). The Case for Regional Development Intervention: Place-Based Based versus PlaceNeutral Approaches.

Berdegué, J. y Proctor, F. (2014). Cities in the Rural Transformation. Working Paper Series. Working Group: Development with Territorial Cohesion, (122).

Ciccone, A. (2002). Agglomeration effects in Europe. European Economic Review, pp. 213-227.

Figueroa, O. y Rozas, P. (2005). Conectividad, ámbitos de impacto y desarrollo territorial: el ca- so de Chile. SERIE Recursos naturales e infraestructura.

Graham, D. J. (2007). Agglomeration, productivity and transport investment. Journal of Transport Economics and Policy, 41:317-343.

Joignaux, G. y Langumier, J.-F. (2004). Les observatoires autoroutiers: L'expérience des autoroutes Paris-Rhin-Rhône et quelques enseignements. Les Cahiers Scientifiques du Transport, (46):324.

Kernohan, D. y Rognlien, L. (2011). Wider economic impacts of transport investments in New Zealand.

Langumier, J.-F. (2002). Pourquoi des observatoires autoroutiers et pour qui?/ Motorway observation units: why create them and for whom? Géocarrefour. Autoroutes, acteurs et dynamiques territoriales., 77(1):95-97.

Litman, T. (2014). Evaluating Accessibility for Transportation Planning.

Mackie, P. y Worsley, T. (2013). International comparisions of transport appraisal practice.

Ministerio de Obras Públicas (2004). Análisis de accesibilidad territorial. Fronteras interiores.

Ministerio de Obras Públicas (2014). Manual de Carreteras. Dirección de Vialidad. Ministerio de Obras Públicas, Santiago, Chile.

Moreno, R. y López-Bazo, E. (2003). The impact of infraestructure on regional economic growth: Some results on its spillover effect.

OCDE (2002). Impact of transport infraestrcuture investment on regional development.

Ortúzar, J. y Willumsen, L. (2008). Modelos de transporte. Ediciones de la Universidad de Cantabria, Santander.

Rimisp (2013). Pobreza y desigualdad - Informe Latinoamericano 2013 - Empleo de calidad y territorio.

Subsecretaría de Desarrollo Regional y Administrativo (2009). Informe de desarrollo territorial sobre Chile - Síntesis descriptiva.

Subsecretaría de Desarrollo Regional y Administrativo (2013). Estudio Identificación de localidades en condición de aislamiento 2012.

Vickerman, R. (2007). Recent evolution of research into the wider economic benefits of transport infraestructure investments.

Rev. Est. de Políticas Públicas, 2016, vol. 3 (junio) 